\title{
Duodenal ulceration into the cystic artery
}

\author{
G.A. Ford ${ }^{1}$, A.H.R.W. Simpson ${ }^{2}$, M.W.L. Gear ${ }^{2}$ and S.P. Wilkinson ${ }^{1}$ \\ Departments of ${ }^{1}$ Medicine and ${ }^{2}$ Surgery, Gloucestershire Royal Hospital, Great Western Road, Gloucester \\ GL1 $3 N N, U K$.
}

\begin{abstract}
Summary: We report two cases of massive gastrointestinal bleeding due to anterior duodenal ulceration into the cystic artery, with gallbladder infarction as a complication. These cases indicate the potential dangers of laser therapy or embolization in bleeding anterior duodenal ulcers penetrating the cystic artery, as such therapy will arrest blood flow through the cystic artery and may precipitate gallbladder infarction.
\end{abstract}

\section{Introduction}

Standard surgical texts state that anterior duodenal ulcers when complicated tend to perforate whereas posterior ulcers, particularly post-bulbar ulcers, tend to bleed because of the underlying vascular bed. ${ }^{1}$ Anterior duodenal ulceration into the cystic artery is rare; to our knowledge only four cases have previously been reported..$^{2-4}$

\section{Case reports}

\section{Case 1}

An 82 year old woman was admitted with haematemesis and melaena. She was taking diclofenac, a non-steroidal anti-inflammatory drug, for osteoarthritis. Hypotension was present on admission but her condition stabilized after transfusion of 5 units of whole blood. Abdominal examination was normal. Upper gastrointestinal endoscopy demonstrated an $8 \mathrm{~mm}$ diameter anterior duodenal ulcer with a visible vessel and adherent clot. A persisting pyrexia of $39^{\circ} \mathrm{C}$ and peripheral neutrophilia were attributed to a urinary tract infection and treatment with intravenous ampicillin was commenced. Forty eight hours after admission she had a large rebleed. At laparotomy a large duodenal ulcer was found completely penetrating the anterior duodenal wall. An inflammatory mass involving the gallbladder sealed the defect. The bleeding vessel in the base of the ulcer was the cystic artery and the gallbladder showed patches of early gangrene. There were no gallstones. Cholecystectomy, truncal vagotomy and closure of the perfora-

Correspondence: S.P. Wilkinson, M.D., F.R.C.P., Gloucestershire Royal Hospital, Great West Road, Gloucester GL1 3NN, UK.

Accepted: 20 July 1989 tion with an omental patch were performed. Histology confirmed the laparotomy findings with the cystic artery in the base of the ulcer partially occluded by thrombus. There were no gastrointestinal complications following surgery and the patient made a good recovery.

\section{Case 2}

A 77 year old man was admitted with an ischaemic left leg. He underwent left femoral embolectomy and was anticoagulated with heparin then warfarin. He gave a history of mild epigastric pain. Six days later haematemesis and melaena occurred. Abdominal examination was normal. After transfusion of 7 units of blood, laparotomy was performed. A full thickness anterior duodenal ulcer was found walled off by the gallbladder which was small and atrophic. The bleeding vessel in the base of the ulcer was the cystic artery or a large branch of the artery. No gallstones were present. The shrunken gallbladder was separated from the duodenum and left in situ, and the bleeding vessel oversewn. The duodenal defect was closed with an omental patch. There were no post-operative complications.

\section{Discussion}

Duodenal ulcer haemorrhage is the commonest cause of massive arterial bleeding from the gastrointestinal tract, usually originating from branches of the pancreatico-duodenal artery. ${ }^{5}$ Despite the frequency of duodenal ulceration, there are few studies examining the relationship of the site of ulceration to complications. Studies of the location of uncomplicated duodenal ulceration suggest that anterior ulcers are twice as common as posterior ulcers and that ulcers are post bulbar in $5 \%$ of 
cases. ${ }^{6-8}$ Although it is stated in textbooks that posterior duodenal ulcers are complicated more frequently by haemorrhage, few surgical or endoscopic series have specifically examined this question. ${ }^{1}$ In one series $38 \%$ of endoscopically diagnosed posterior duodenal ulcers presented with haemorrhage as compared to $25 \%$ of anterior duodenal ulcers. ${ }^{6}$ Because of the higher incidence of anterior duodenal ulcers in this series, however, cases of anterior ulcer haemorrhage were more frequent than posterior ulcer haemorrhage. This contradicts standard teaching, although it may still be the case that massive haemorrhage is a more frequent complication with posterior duodenal ulcers.

The first part of the duodenum has as its immediate anatomical relations, the head of the pancreas posteriorly, and the liver and gallbladder anteriorly. Ulceration of anterior duodenal ulcers into the gallbladder and liver is well described.9,10 Direct erosion of duodenal ulcers into the cystic artery is very uncommon. Postolov and Ziubina report a case of gangrenous cholecystitis in association with massive bleeding from a peptic ulcer. ${ }^{2}$ The exact site of the ulcer is not clear, in a translation of this Russian paper, but it is probable that the ulcer was anterior and had penetrated into the cystic artery. Glazer $e t$ al. describe penetration of a lateral post-bulbar duodenal ulcer into the gallbladder and erosion of the branch of the cystic artery leading to massive gastrointestinal haemorrhage in one patient. ${ }^{3}$ In a second patient they describe penetration of a post-bulbar posterolateral duodenal ulcer into a previously ligated cystic artery stump, the patient having undergone cholecystectomy 7 years previously. Ring et al. describe penetration of a branch of the cystic artery by an ulcer in the second part of the duodenum. ${ }^{4}$ Further anatomical localization of the ulcer is not given in the latter case.

Because the cystic artery is an end artery, obstruction of blood flow may cause gallbladder infarction. Gallbladder ischaemia and infarction are usually associated with gallstones, and gallbladder infarction in their absence is unusual. ${ }^{11}$ In addition to the one case with duodenal ulceration into the cystic artery mentioned, it has been described as a complication of atherosclerosis affecting the cystic artery, extension of a dissecting aortic aneurysm into the common hepatic artery, a coeliac artery embolus, coeliac angiography, therapeutic arterial embolization of a bleeding duodenal ulcer (not penetrating the cystic artery) and polyarteritis nodosa of the gallbladder. ${ }^{12-16}$
Our cases and those previously discussed, suggest that penetration of the cystic artery or its branches may occur rarely with anterior or lateral post-bulbar duodenal ulcers. Such a complication with posterior duodenal ulcers would be extremely unlikely because of the anatomical relationships discussed. In our first patient, erosion of the cystic artery was probably an acute occurrence and subsequent ischaemia caused gallbladder infarction. In our second patient, the ulcer may have been chronic and a longstanding endarteritis of the cystic artery resulted in chronic ischaemic atrophy of the gallbladder. Gallbladder infarction was not precipitated in our second case treated with undersewing of the bleeding artery, probably because the gallbladder was atrophic and fibrotic with a diminished blood flow requirement. There may also have been blood flow to the gallbladder from other smaller proximal branches of the cystic artery. In the case described by Ring et al. ligation of the bleeding branch of the cystic artery without cholecystectomy did not precipitate clinically apparent gallbladder infarction. Caution would suggest that when massive bleeding occurs from the cystic artery or a major branch of the artery supplying a normal gallbladder, cholecystectomy should be performed, as simple undersewing of the artery could reduce blood flow through the cystic artery and precipitate gallbladder infarction.

The cases described have important implications for the management by physicians, surgeons or radiologists of bleeding anterior or post-bulbar duodenal ulcers. Gastrointestinal haemorrhage from peptic ulceration is increasingly being treated by electrocoagulation or laser therapy where blood flow through the bleeding vessel in the base of the ulcer is arrested by coagulation of the bleeding vessel distally and proximally. In the light of the literature discussed, it is likely that if embolization or endoscopic means were used to arrest haemorrhage from the cystic artery, gallbladder infarction would occur.

Penetration of the cystic artery should be considered with massive bleeding from anterior or post-bulbar duodenal ulcers. The possibility of gallbladder infarction should be considered if pyrexia or other suggestive clinical signs occur. If duodenal ulceration into the cystic artery is associated with massive haemorrhage, our cases and those reported in the literature suggest that the treatment of choice is likely to be surgical intervention with oversewing of the ulcer, cystic artery ligation and cholecystectomy rather than endoscopic intervention or therapeutic embolization. 


\section{References}

1. Dudley, H.A.F. Acute bleeding from the upper gastrointestinal tract: In: Dudley, H.A.F. (ed.). Emergency Surgery, 10th ed. J. Wright \& Sons, Bristol, 1977, pp. 397-408.

2. Postolov, P.M. \& Ziubina, E.N. Combined profuse peptic hemorrhage and gangrenous cholecystitis. Vestn Khir 1985 , 135: 63-64.

3. Glazer, H.B., Stanley, R.J. \& Koshler, R.E. Cystic artery haemorrhage. A complication of penetrating duodenal ulcer. Radiology 1980, 136: 623-625.

4. Ring, S.M., Beranbaum, E.R., Madayag, M.A. et al. Peptic erosion of the cystic artery. An unusual etiology of spontaneous gastrointestinal hemorrhage. Angiology 1972, 23: 554-557.

5. Palmer, E. Diagnostic experiences with 1000 upper gastrointestinal bleeders. RI Med J 1964, 47: 229-231.

6. Kang, J.Y., Nasiry, R., Guan, R. et al. Influence of the site of a duodenal ulcer on its mode of presentation. Gastroenterology 1986, 90: 1874-1876.

7. Kirk, R.M. Site and localization of duodenal ulcers: a study at operation. Gut 1968, 9: 414-419.

8. Al-Bahrani, Z.R., Kassir, Z.A. \& Al-Doree, W. The location and multiplicity of chronic duodenal ulcer. Gastroenterol Jpn 1980, 15: 539-542.
9. Allard, J.C. \& Kuligowska, E. Percutaneous treatment of an intrahepatic abscess caused by a penetrating duodenal ulcer. $J$ Clin Gastroenterol 1987, 9: 603-606.

10. Michowitz, M., Farago, C., Lazarovici, I., Solowiejczjk. Choledochoduodenal fistula: a rare complication of duodenal ulcer. Am J Gastroenterol 1984, 79: 416-420.

11. Matz, L.R. \& Lawrence-Brown, M.M.D. Ischaemic cholecystitis and infarction of the gallbladder. Aust NZ J Surg 1982, 52: 466-471.

12. Dillard, B.M. \& Black, W.C. Polyarteritis nodosa of the gallbladder and bile ducts. Am Surg 1970, 36: 423-427.

13. Rosen, Y. \& Chen, C. Infarction of the gallbladder - a complication of hypertension. Am J Gastroenterol 1977, 67: 249-252.

14. Jacob, E.T., Shapira, Z., Morag, B. \& Rubinstein, Z. Hepatic infarction and gallbladder necrosis complicating arterial embolization for bleeding duodenal ulcer. Dig Dis Sci 1979, 24: 482-484.

15. Eastland, M.E. \& Schwartz, S.I. Infarction of the gallbladder after celiac arteriography. Am J Surg 1971, 122: 558-559.

16. Hawe, A. Coeliac artery embolus presenting with gangrene of the gallbladder. Br J Clin Pract 1967, 21: 557-558. 